\title{
Luchas de auto-afirmación disciplinar en la universidad francesa de la Tercera República: el debate Simiand-Seignobos
}

Struggles of disciplinary affirmation at the French university of
the Third Republic: the Simiand-Seignobos debate

David J. Domínguez GonzÁlez

Universidad Complutense de Madrid dadomi01@ucm.es (ESPAÑA)

Recibido: 08.112018

Aceptado: 20.09.2019

\section{RESUMEN}

En este artículo revisaremos el clásico debate que enfrentó a Simiand y Seignobos en la primera década del siglo XX. Por lo general, la imagen oficial del asunto retiene sólo el aspecto epistemológico de la disputa. Se recuerda a esta última por la crítica de Simiand a los determinismos implícitos (ídolo político, individual, cronológico) en el método de los historiadores. Lo que se propone, sin embargo, en este texto es un cambio de mirada en la comprensión de esta disputa intelectual. Lejos de agotar el interés en la confrontación sobre las prácticas científicas normales, se procederá a insertar la disputa en el marco de una lucha más amplia que opone dos candidaturas (sociología e historia) a la hegemonía de las ciencias humanas de la universidad francesa. Para ello es preciso analizar qué margen de cobertura institucional detentaba en ese momento la disciplina histórica y la sociología en el marco de la enseñanza superior republicana. Por último, se reflexiona sobre la falta de simetría que caracterizó a la relación entre ambas disciplinas, para concluir que tal disparidad quizá sea la causa del semifracaso del ataque durkheimiano frente a los historiadores.

\section{PALABRAS CLAVE}

Sociología, reflexividad, sociología de los intelectuales. 


\begin{abstract}
In this article we will review the classic(well-known/traditional) debate faced by Simiand and Seignobos in the first decade of the 20th century. Generally, the epistemological aspect of the dispute is considered the main matter at stake. The debate is usually remembered due to Simiand's critique on the implicit determinisms (political, individual, chronological idol) in the historians's method. Nevertheless, the purpose of this article is to suggest a change of look in understanding this intellectual dispute. Far from exhausting the discussion on normal scientific practices' confrontation, the dispute will be studied within the framework of a broader struggle in which two candidatures (sociology and history) strives for the hegemony of the human sciences of the French university. To that end, it is necessary to analyze which institutional spaces were respectively filled by the historical and by the sociological discipline within the framework of the republican higher education. Finally, we will reflect on the lack of symmetry that characterized the relationship between both disciplines, to conclude that such disparity may be the cause of the semi-failure of the Durkheimian attack against the historians.
\end{abstract}

\title{
KEY WORDS
}

Sociology, reflexivity, sociology of intellectuals.

\section{INTRODUCCIÓN Y PRESENTACIÓN DEL TEMA}

En 1903, el sociólogo francés François Simiand imparte una polémica conferencia en contra de los fundamentos del método historiográfico. En ella arremete contra los tres ídolos que, a su parecer, impedían la conversión de la ciencia histórica en un saber científico. El método histórico, presentado hasta ese momento con pompa y boato en la universidad de la Tercera República, era cuestionado por un sociólogo que apenas superaba los treinta años de edad. Los historiadores habían sido interpelados a través de la crítica de uno de sus más eminentes representantes, Charles Seignobos. Tal ataque, sin embargo, no encontró una respuesta inmediata de su parte, si bien es cierto que las críticas posteriores de la sociología durkheimiana acabaron por motivar la respuesta del historiador y la posibilidad de entablar un debate conjunto ${ }^{1}$.

${ }^{1}$ La polémica se compone de las dos célebres conferencias pronunciadas por Simiand (Método histórico y ciencia social, de 1903, y La causalidad en historia, de 1906); de la réplica de Seignobos (Las condiciones prácticas de la búsqueda de las causas en el trabajo histórico, de 1907); y, por último, de una conferencia (Lo desconocido y lo inconsciente en historia, de 1908) en la que Durkheim y Seignobos debaten acerca del papel de las representaciones colectivas en los fenómenos sociales 
El presente artículo trata de retomar este debate. Pero lo hace, eso sí, de un modo diferente a como se ha realizado con antelación. ${ }^{2}$. Por lo general, la imagen oficial del asunto retiene tan sólo un aspecto restringido de la disputa. Se recuerda a esta última por la crítica de F. Simiand a los tres obstáculos que impiden la conversión de la ciencia histórica en un terreno próximo a las problemáticas sociológicas. La polémica es de sobra conocida: crítica del ídolo político, del ídolo individual y del ídolo cronológico. Sin embargo, este tipo de lectura, centrada en la disputa sobre las prácticas científicas normales, no resume por entero un episodio del que se pueden extraer múltiples desafíos disciplinares (Besnard 1986: 32).

De ahí la necesidad de un estudio sobre la dimensión institucional del debate: sin él se corre el riesgo de limitar la mirada a los argumentos ofrecidos por los autores, dejando de lado, por razones analíticas, el estudio de las luchas y las estrategias de auto-afirmación disciplinar que acompañan al proceso de disciplinarización de los saberes en el marco de la universidad republicana. El estudio que se presenta aquí tratará de poner al descubierto las condiciones institucionales en las que se desplegó la polémica. En efecto, ¿qué posición ocupaba la ciencia histórica y la sociología en el seno de la universidad republicana? ¿Qué lógicas y qué estrategias disciplinares están en juego en el momento en que Simiand pronuncia su conferencia en la Société d'Histoire Moderne? ¿Qué hay detrás del debate sobre las prácticas científicas normales?

Para responder a estas cuestiones hemos de partir del supuesto de que la disputa posee varios niveles de lectura: en apariencia se trata sólo de un debate metodológico, pero por debajo o por mediación de éste se perfila otra batalla de naturaleza institucional que opone dos candidaturas a la hegemonía del campo de las ciencias humanas en la Tercera República francesa (Réberioux 1983: 227).

El objetivo de las páginas siguientes consistirá en precisar las condiciones reales de los dos enclaves disciplinares, a fin de poner al descubierto la relación asimétrica, profundamente desigual, que mantuvo la sociología durkheimiana con la infraestructura institucional del saber histórico en la universidad de la Tercera República.

\section{UNA DISPARIDAD MANIFIESTA: LA HISTORIA Y LA SOCIOLOGÍA EN LA UNIVERSIDAD DE LA TERCERA REPÚBLICA}

Cuando se piensa en algún detalle que pueda caracterizar el debate, es casi seguro que será la polémica y el tono controvertido el que acabe imponiéndose. El debate, se dirá, es un asunto alentado por un sociólogo al que no le importan las etiquetas ni las formas convencionales de cortesía intelectual. Sin duda, el discurso de Simiand no está exento de tales imputaciones. No es difícil percibir en él un tono polémico y un mensaje poco condescendiente con los marcos cla-

${ }^{2}$ Véanse los trabajos de Devoto (1992), Noiriel (2003), García (2007), Revel (1999). 
sificatorios y las formas de inferencia de los historiadores universitarios. Esto es cierto, pero quedarse ahí anularía la posibilidad de plantear una lectura más histórica del debate. En efecto, ¿por qué un tono tan enérgico en el debate? ¿Por qué no disimular simplemente la radicalidad de la crítica mediante un lenguaje cortés y los eufemismos de los intercambios intelectuales? ¿Qué indica pues la virulencia del ataque? ¿Forma parte de la psicología subjetiva del sociólogo o se puede atisbar otra clave de análisis?

La respuesta a estas preguntas pasa por el estudio de las condiciones institucionales en las que se encuentra la enseñanza de la historia y la sociología en la universidad francesa de la Tercera República. Empezaremos por la ciencia histórica. ¿Qué extensión ocupa en el contexto de la universidad francesa de comienzos del siglo XX? ¿Qué transformaciones trajeron consigo las reformas republicanas?

\subsection{La historia, hermana mayor...}

Para responder a tales cuestiones la exposición ha de organizarse a partir de dos cuestiones: la primera consistirá en enumerar las medidas más relevantes de las reformas republicanas, tanto en el marco general de la enseñanza universitaria como en el caso específico de la enseñanza histórica. Y la segunda tratará de centrarse en los efectos de las reformas para la autonomización profesional de la historia.

1/ Comencemos por las medidas concretas. En líneas generales se pueden dividir en dos grandes bloques:

Por un lado, un bloque (a) centrado en las disposiciones orientadas a mejorar la dotación económica de las facultades, siendo reseñable la mejora que tuvo lugar en las facultades de Letras y de Ciencias.

Y por otro, un bloque (b) centrado en las medidas que modificaron el régimen interno de los estudios universitarios. En este punto nos centraremos en aquellos aspectos que plantean una diversificación de las disciplinas practicadas, a fin de ver que tales medidas van generando un espacio -a la vez cognitivo e institucional- en el que la ciencia histórica empieza a cobrar autonomía.

En cuanto al primer bloque (a), cabe recordar el incremento generalizado de las dotaciones económicas ${ }^{3}$. Este fenómeno trajo consigo cambios sustanciales

${ }^{3}$ En 1875 las facultades obtuvieron un presupuesto de 5,1 millones de francos, mientras que los liceos y los collèges alcanzaron los 3,9 millones, es decir un 25\% del presupuesto total del Ministerio. Desde 1878 estas cifras se dispararon: ese mismo año la dotación presupuestada para las universidades alcanza los 9,2 millones, mientras que los liceos y collèges obtienen 5,2 millones, es decir un 26,7\% del presupuesto total. En 1890 la dotación de las facultades asciende a 11 millones, mientras que la educación secundaria sobrepasaba los 15 millones, un 18,7\% del presupuesto total para ese año. De todo ello se deducen dos conclusiones: la primera es que la educación primaria (ausente en estas cifras) aglutinaba la mayor parte de las dotaciones del Ministerio, dado que se trataba de una red universal, obligatoria y gratuita. Y la segunda, es el aumento de los fondos desti- 
en la educación universitaria. Cambios que iban desde la mejora de la infraestructura material (bibliotecas, laboratorios, aulas) hasta el incremento de cátedras y plazas universitarias. De igual modo, se puede incluir la creación de un sistema de becas orientado a 'producir' un público universitario 'profesional', alejado del perfil académico que reproducían las viejas facultades napoleónicas, con sus cursos abiertos y su afición a los discursos solemnes en grandes auditorios públicos (Keylor 1975: 62).

La creación del sistema de becas es, en este sentido, la pieza maestra de todo el cúmulo de reformas universitarias. Primero en 1877, con la dotación anual de trescientas becas (bourses d'études) para los estudiantes de licence. Y después, en 1880, con la implementación de doscientas becas más destinadas a los alumnos que preparaban la agrégation (Gérard en Amalvi et al. 2005: 266). En ambos casos se advierte un primer paso en la política de promoción universitaria, sobre todo en el sector de las facultades provinciales ${ }^{4}$. El objetivo era claro: se trataba de incentivar la demanda de los estudios en letras y en ciencias ${ }^{5}$, algo perfectamente comprensible si se tiene en cuenta el escaso número de alumnos que poblaban las facultades del sistema napoleónico ${ }^{6}$. Ahora bien, para poner en marcha un orden de enseñanza superior había que producir al alumnado como alumnado. Las becas constituyen un primer paso en esa dirección. Pero junto a ello, y de manera no menos importante, se atisba también una política basada en la transformación del cuerpo profesoral ${ }^{7}$. Los datos no dejan lugar a dudas ${ }^{8}$, si bien es cierto que un crecimiento como éste se debe por un lado a la ampliación de cátedras existentes y a la promoción de una figura novedosa en las facultades francesas, los maîtres de conférences. Especialmente significativo es el incre-

nados a la enseñanza superior, que constituía una importante apuesta del Ministerio de Instrucción pública. Más datos en Karady (1983: 95)

${ }^{4}$ Véase el decreto que regula la dotación de becas para la licence. Dice así: "2. La mayoría de esas becas serán atribuidas a las facultades de los departamentos" ("Arreté concernant les bourses de Faculté. 5 Novembre 1877' en Beauchamp, 1880: 169). A este respecto cabe señalar que entre los años 1879-1892 el 72\% de las becas de licence se destinaron a las facultades provinciales. Véase Karady (1983: 97).

5 " $4^{\circ}$ Las facultades de medicina y las escuelas superiores de farmacia podrán obtenerlas, pero en menor medida que las facultades de ciencias y de letras" ("Arreté concernant les bourses de Faculté. 5 Novembre 1877" en Beauchamp 1880: 169).

${ }^{6}$ En la época del Segundo Imperio la demanda de educación universitaria era bastante inferior a la oferta de plazas existentes. En este sentido, las políticas de crecimiento universitario no se deben a un aumento real de la demanda, sino a una voluntad expresa de los republicanos moderados, que veían en ello una herramienta contra la enseñanza eclesiástica. Véase Karady (1983: 91).

7 De ahí la financiación por parte del gobierno a las jóvenes promesas de la intelectualidad francesa. En aquel momento (1860-1880) realizar una estancia de investigación en el país germano era sinónimo de rigor científico e independencia de espíritu. El caso de Gabriel Monod (1897: 100102) es claro al respecto, y lo mismo cabe decir de Ernest Lavisse (1885: 228-229). Sin embargo, testimonios posteriores como el de Seignobos (1881: 565-566) ofrecen una perspectiva menos condescendiente de las universidades alemanas. Un análisis detallado de tales informes en Charle (1994: 21-131).

${ }^{8}$ En términos globales, Antoine Prost (1968: 234) ofrece cifras significativas. Así, de 503 profesores en 1880 se pasa a 650 en 1890, y a 1048 en 1909, lo que supone más del doble de los efectivos enseñantes universitarios. 
mento de cátedras y plazas relacionadas con la ciencia histórica. Es ahí donde se percibe una mayor insistencia por parte de las instituciones, como si el apoyo y la participación activa de los historiadores en las reformas ${ }^{9}$ se tradujesen en una mayor dotación para el desarrollo de la especialización histórica. Los ejemplos son múltiples: en el Collège de France el número de cátedras relacionadas con temáticas 'históricas' pasa de tres a quince. Algo similar ocurre en la EPHE, que de dos cátedras existentes en 1869 (Gabriel Monod y Alfred Rambaud, este último convertido después en ministro) se pasan a siete en 1897 (Gérard en Amalvi et al. 2005: 277). Sin embargo, el incremento más claro se centra en la red de facultades de letras. Es aquí donde se produce un incremento inusitado, llegando en algunos casos a transformar el cuerpo profesoral y generar un elenco de especialidades que pocos años antes hubiera sido impensable. En la Sorbona, por ejemplo, la proliferación de cátedras históricas empieza a ser habitual: surgen primero las especializadas por épocas históricas, como la Edad Media (Fustel de Coulanges, en 1875), la Historia Moderna y Contemporánea (Alfred Rambaud, en 1875) o la Revolución francesa (Alphonse Aulard, en 1885). Después, la enseñanza irá especializándose más, al punto de surgir nuevas cátedras centradas en cuestiones de 'metodología histórica' (Ch. Seignobos, en 1907) o en la transmisión de 'ciencias auxiliares' (Ch. V. Langlois, en 1907) ${ }^{10}$, lo cual revelaba un grado de división interna del trabajo y de especialización técnica.

En apenas treinta años se había triplicado el número de cátedras asociadas a temas 'históricos'. Se pasó, en concreto, de diecisiete en 1876 a cincuenta y siete en 1910 (Ibíd: 278). Una cifra que por sí sola no tiene valor representativo, pero que si se compara con el número total de cátedras en las facultades de letras nos deja un valor harto significativo: es decir, una representación que alcanza en 1910 el 33\% de las cátedras existentes, lo que demuestra una posición más que relevante para la disciplina histórica (Ibíd: 279). Una evolución similar se revela en los datos que suministra Ferdinand Lot (1904: 115-116) en su análisis de las facultades de letras en Francia y Alemania. Ciñéndose al periodo que va de 1895 a 1905, se percibe un incremento general en el número de docentes (no necesariamente cátedras) dedicados a la enseñanza histórica, y ello en todos los espacios de la educación superior francesa. Asimismo, es palpable la existencia de una cierta (aunque escasa) especialización en las posiciones docentes en historia (Ibídem). Contrasta sin embargo la clara desventaja de Francia con respecto a Alemania, que sigue detentando de lejos la primacía en el ámbito de la enseñanza histórica universitaria, aunque cada vez de manera menos indiscutible.

${ }^{9}$ Recuérdese el peso que tuvieron los historiadores (Ernest Renan, HippolyteTaine, Monod, Fustel de Coulanges, Lavisse) en la Société pour l'étude des questions d'enseignement superieure.

${ }^{10}$ Posteriormente, este mismo proceso se desarrolla en las facultades provinciales. Véase Gerard (en Amalvi et al. 2005: 278). 
Aumento en el número de posiciones docentes en historia. Universidades francesas y alemanas. Periodo 1895-1896 a 1904-1905

\begin{tabular}{|l|c|c|}
\hline Fuente: F. Lot (1904: 115-116) & $1895-1896$ & $1904-1905$ \\
\hline $\begin{array}{l}\text { Instituciones parisinas (Sorbona, ENS, École des } \\
\text { Chartes, EPHE, College de France) }\end{array}$ & 21 & 29 \\
\hline Universidades provinciales francesas & 36 & 45 \\
\hline Total de universidades francesas & 57 & 74 \\
\hline Total universidades alemanas & 134 & 152 \\
\hline
\end{tabular}

Posiciones docentes en historia. Universidades francesas y alemanas. 1904-1905

\begin{tabular}{|l|c|c|c|c|}
\hline Categoría & París & $\begin{array}{c}\text { Universidades } \\
\text { provinciales } \\
\text { francesas }\end{array}$ & $\begin{array}{c}\text { Puestos totales de } \\
\text { las universidades } \\
\text { francesas }\end{array}$ & $\begin{array}{c}\text { Universidades } \\
\text { alemanas }\end{array}$ \\
\hline Historia Antigua & 9 & 8 & 17 & 33 \\
\hline Historia Medieval & 13 & 12 & 25 & 44 \\
\hline Historia Moderna & 7 & 13 & 20 & 29 \\
\hline $\begin{array}{l}\text { M e d i e v a l - } \\
\text { Moderna }\end{array}$ & - & - & - & 11 \\
\hline Historia local, etc & - & 12 & 12 & $\mathbf{1 5 2}$ \\
\hline Total & $\mathbf{2 9}$ & $\mathbf{4 5}$ & $\mathbf{7 4}$ & 35 \\
\hline
\end{tabular}

Fuente: F. Lot (1904: 115-116)

En cuanto al segundo bloque (b), cabe señalar los siguientes aspectos:

Comenzaremos por una medida importante: la ley del 25 de diciembre de 1880, según la cual el Ministerio de Instrucción Pública ordena modificar las condiciones para obtener la licence es lettres. Influido por los informes de las facultades de letras ${ }^{11}$, el Ministerio decide introducir una primera forma de especialización académica. Para ello implementa esta medida legislativa, según la cual se precisan dos pruebas objetivas para aquellos que pretendan obtener la $l i$ cence es lettres. La primera de ellas consiste en una prueba común, basada en la

11 Véanse las 'Réponses des Facultes et des Conseils académiques sur les changements à apporter à l'examen de la licence es lettres', de Michel Bréal (en Beauchamp 1880: 526-528). 
producción escrita de dos textos en francés y en latín; la segunda, en cambio, se trataba de una prueba especial, que consistía en realizar una composición escrita acerca de un tema que tuviera cabida entre las materias posibles (filosofía, letras o historia $)^{12}$. Esta medida, aunque insuficiente, constituye un primer paso en el camino de la consolidación del itinerario académico del saber histórico.

Convertidos en una licencia mixta, los estudios en letras implementan un cierto grado de especialización académica; lo básico, como diría Lavisse, para que la nueva licence es lettres, o licencia mixta, como se prefiera, pueda entenderse como el acta de nacimiento del 'estudiante de historia'. Dicho de otro modo, a partir de ahora se sientan las bases para que los alumnos de la École nórmale empiecen a copar los puestos especializados en las distintas facultades de letras. Es ahora cuando se crean múltiples plazas y cátedras profesorales, lo cual permite a los estudiantes en letras adquirir una cultura disciplinar específica, fuese en filosofía, en letras o en historia.

Junto a ello cabe mencionar la implantación de las conférences. Instituidas por el decreto del 5 de noviembre de $1877^{13}$, se dirigían a paliar las deficiencias del sistema napoleónico de transmisión del saber. Así, a diferencia de las clases magistrales ante un público heterogéneo, las conférences se caracterizaban por su intimidad y su inequívoca vocación metodológica. Se trataba, en definitiva, de cursos dirigidos a un grupo reducido de personas, lo que las convertía en un espacio estratégico para fomentar el aprendizaje de la metodología (ciencias auxiliares) y el desarrollo de los trabajos dirigidos ${ }^{14}$. Tales cursos, por su parte, eran confiados a los maîtres de conférences, una figura de origen normalien que comenzó a generalizarse en las facultades públicas en el decenio de 1880, convirtiéndose en pocos años en un agente modernizador de la enseñanza universitaria (Mayeur en Amalvi et al, 2005: 15). Los maîtres de conférences se reclutaban entre los agregados y los doctores, lo que hacía de ellos un cuerpo profesoral joven e influenciado (cuando no formado) por las innovaciones pedagógicas que estaban teniendo lugar en las universidades europeas ${ }^{15}$. Es aquí, en cierto modo, donde se puede encontrar la traducción francesa de los viejos seminarios organizados en las universidades alemanas. El carácter íntimo y práctico que había caracterizado a estos últimos ${ }^{16}$, parece retomarse mediante

${ }^{12}$ Véase el 'Décret portant modification des épreuves de la licence es lettres. 25 décembre 1880', en Ibíd: 528-530).

13 Véase el 'Arreté concernant les conférences instituées dans les établissements d'enseignement supérieur. 5 de novembre 1877' en Beauchamp (1880: 166-167).

14 "Art. 4. Las conferencias de todo tipo estarán necesariamente acompañadas de cuestiones dirigidas por el profesor a los alumnos, o intercambios entre los alumnos bajo su dirección (...)" (Ibíd: 168-169)

15 El decreto del 5 de noviembre de 1877 fija incluso las preferencias en cuanto al criterio de reclutamiento de los maîtres de conférences para las Facultades de letras. Véase Beauchamp (1880: 169).

${ }^{16}$ Una descripción del tipo de pedagogía utilizada en los seminarios de las universidades alemanas se puede encontrar en los informes de los historiadores franceses del momento, especialmente en Monod (1897: 100-103) y Seignobos (1881: 565-578), este último en un tono más crítico pero muy descriptivo en cuanto a la naturaleza filológica de los seminarios en la década de 1880 . 
una pedagogía basada en la interacción directa entre alumno y profesor. De ese modo, se conseguía un doble objetivo, al menos en la formación de historiador: por un lado, afianzar el saber técnico y metodológico de la disciplina, y por otro, evitar el riesgo, tan común en las facultades napoleónicas, de caer en la afición literaria (Lavisse 1885: 56-57). Tal pedagogía, sin embargo, ya estaba aplicándose en las universidades alemanas desde algunas décadas antes: lo que hacía que sus productos universitarios (diplomas, profesores, revistas universitarias, actas de investigación) adoptasen una posición dominante en el mercado internacional de bienes simbólicos en Letras y en Ciencias (Karady en Charle et al, 1985: 38). Conscientes de esta hegemonía, la élite de los universitarios franceses (organizados en comisiones pro-reforma ${ }^{17}$ ) trataron de importar el modelo pedagógico que hacía posible esos resultados. Para ellos el modelo iniciado por sus vecinos germanos representaba un estadio superior de la enseñanza universitaria ${ }^{18}$. Cansados de un modelo trasnochado, en el que las decisiones políticas pesaban sobre las nominaciones de los profesores y el contenido de los programas, los intelectuales franceses veían al otro lado del Rin un modelo al cual imitar, un modelo a la vez libre y organizado (Lehrfreiheit und Lernfreiheit) ${ }^{19}$. Libre porque se trataba de un espacio caracterizado por la movilidad y la descentralización universitaria, en el cual no existía un único centro catalizador (al estilo de la Sorbona, por ejemplo) que aglutinase la mayoría de los recursos técnicos (laboratorios, bibliotecas) y humanos (profesores, cátedras, etc.). Y organizado porque a pesar de no tener una fisonomía centralizada el modelo aseguraba la constitución de un mercado académico progresivamente estandarizado, en el que la formalización y la circulación de los saberes aseguraba el desarrollo de la innovación y la mutación disciplinar (García en Delacroix et al: 2007: 105). Es así como se entiende por ejemplo la financiación de viajes a las universidades alemanas, una tradición relativamente arraigada en la élite de los profesores parisinos ${ }^{20}$. Y es así como

17 Algunos de los intelectuales y parlamentarios más relevantes formaron parte de grupos y comisiones donde se debatían propuestas pedagógicas en vistas a las reformas universitarias. El caso más relevante es la Société pour l'étude des questions d'enseignement supérieur, de la que formaban parte Pasteur, Berthélot, Waddington, Dumont, Renan, Taine, Monod, Lavisse o el propio Fustel de Coulanges. Entre sus tareas más relevantes: entablar comunicación con las universidades extranjeras, a fin de conocer las innovaciones que se estaban produciendo en materia de organización pedagógica, y poder así trasladar aquellos datos que pudieran despertar algún interés con vistas a un proyecto global de reforma universitaria. Véase al respecto el primer número de la Revue internationale de l'enseignement (1881), órgano de expresión de la Société.

18 Para los republicanos franceses, la derrota ante los ejércitos prusianos (1871) puso de manifiesto el signo de la superioridad intelectual de las universidades germanas. De ahí la importancia de las reformas educativas: los republicanos las concebían como el medio para sentar las bases de la refundación nacional. Sobre este tema, véase Digeon (1959: 365 y ss).

19 Quizá sea entre los historiadores donde esta admiración por la universidad alemana cobra especial relevancia. Véase, a este respecto, las palabras de Monod (1876: 27) y Lavisse (1885: 214).

20 Entre los años 1879 y 1939 cerca del 18\% (es decir 29 de 164) de los profesores de la Sorbona literaria (Historia, Filología, Filosofía, Literatura extranjera) realizaron su estancia de investigación en las universidades alemanas. Nombres como Seignobos, Collignon, Séailles, Durkheim, Camille Jullian, Abel Lefranc son sólo algunos ejemplos de ello. Véase Charle (1994: 27). 
también se entienden las innovaciones (creación de la EPHE) realizadas bajo el auspicio del ministro (e historiador) Victor Duruy, en la época del Segundo Imperio, así como las múltiples reformas que tuvieron lugar en el trascurso de la Tercera República

En este sentido, la implantación de las conférences constituía una medida en clara relación con los seminarios de investigación alemanes (Charle, 1994: 45). El propio Camille Julian había concluido lo mismo en su informe sobre las facultades alemanas, quien acabó reconociendo, no sin ciertas reservas, la superioridad de la universidad alemana frente a las facultades francesas, sobre todo en lo que se refiere a la formación investigadora y los seminarios. Una práctica, como admite el propio historiador, dirigida solamente a una élite, pero una élite mucho más numerosa que la que moraba la École Normale o la EPHE en I 884, pues la organización de los seminarios alemanes aglutinaba un promedio de dos mil o tres mil estudiantes anuales, una cifra más que envidiable si se compara con las pocas decenas que recibían sus cursos en las proximidades del vème arrondissement de París (Jullian, 1884: 422). El objetivo de los reformadores era, por tanto, emular esas cifras por medio de la implementación de las conférences.

En tercer lugar, cabe destacar la creación del diplome d'enseignement supérieur (DES). Este último, ideado por el historiador Ernest Lavisse (1881: 137-151), se inscribe en el marco de la reforma para la agregación en historia y geografía. Instituido el 28 de julio de 1894, el DES contenía una serie de pruebas objetivas cuya superación certificaba el requisito básico para el concurso de agregación en historia. Las pruebas eran además innovadoras: de un lado, la realización (y defensa) de una tesina tutelada, basada en la lectura y el análisis filológico de fuentes originales, y, de otro, la presentación y la defensa de una serie de pruebas orales, consistentes en un análisis crítico de un documento original y en cuestiones relativas a las ciencias auxiliares.

A partir de 1894 el DES se convierte en una condición indispensable para presentarse al concurso de agregación, lo que significa que los profesores de historia pertenecientes a la educación secundaria habrán ejercido, al menos durante un año, tareas relativas a la investigación y las ciencias auxiliares ${ }^{21}$. Poco a poco se va insinuando un itinerario histórico en las facultades de letras. El DES desempeña un papel clave en esta dirección: su énfasis en la comunicación y la dirección tutelada de las tesinas constituye una pieza fundamental en este itinerario, la única, por decirlo así, capaz de asegurar una generación de estudiantes familiarizados con el método histórico y el análisis de fuentes originales, aun cuando no exista (todavía) una licence independiente para la historia.

En la misma línea hemos de comprender la reconstrucción de la Sorbona en 1901. Nacida en un contexto global de reformas, la Nouvelle Sorbonne representa el núcleo duro de los historiadores. Su departamento de historia ha visto doblar sus cátedras entre 1899 y 1910 (Gérard en Amalvi et al. 2005: 288), aglutinando así un cúmulo de recursos que hacían de ella una de las facultades

${ }^{21}$ Véase la 'Circulaire relative à l'agrégation d'histoire. 17 novembre 1894' en Duruy (1902: 72-73). 
más especializadas en saber histórico. Ya se ha señalado el surgimiento de las cátedras de metodología histórica y ciencias auxiliares. Su aparición marca un momento culminante en el desarrollo del itinerario historiográfico. En efecto, al poner el acento en la transmisión y la estandarización de la metodología, la universidad reconoce la especificidad del trabajo histórico como un espacio que debe gozar de autonomía disciplinar. Es ahora cuando la historia disfruta de una presencia notoria en la universidad francesa, cuando empiezan a formalizarse sus protocolos de lectura y se genera una forma estable de sociabilidad académica, donde un conjunto disperso de personas -estudiantes y profesores de distintas universidades- trabaja con métodos y problemáticas comunes, siguiendo normas que plantean una retórica de argumentación similar, condición para el progreso de la propia disciplina pero también para el desacuerdo o el disenso académico ${ }^{22}$.

Por último, ha de señalarse la medida que vino a consagrar el itinerario del saber histórico. Nos referimos al decreto estatuido el 8 de julio de 1907, según el cual la historia (y otras disciplinas) recibe su confirmación definitiva por medio de la creación de una licenciatura independiente ${ }^{23}$. Tal ratificación había sido posible debido a la especialización progresiva del itinerario histórico en los estudios de letras, tal como se ha resumido, pero también era el resultado del enorme influjo que los historiadores franceses habían ejercido en la planificación y posterior aplicación de las reformas universitarias ${ }^{24}$; de hecho, la licence creada en 1907 respondía en realidad a una proposición de la facultad de letras de París, en la cual historiadores como Langlois, Seignobos o Aulard, cuestionaban el peso que seguía teniendo la herencia literaria en las licenciaturas mixtas de 1880 (Gérard en Amalvi et al. 2005: 289). Así pues, la consagración definitiva de los estudios históricos pasaba por la derogación de la licenciatura mixta y la creación de nuevos estudios especializados, cada uno de ellos con sus respectivos objetos de investigación, metodología e itinerario particular. Esta misma sensación es la que se deja entrever en la circular del 31 de octubre de 1907, donde los legisladores presentan la creación de las licenciaturas independientes en clara continuidad con las medidas y los decretos anteriores ${ }^{25}$. En el caso de la historia y de

${ }^{22}$ La existencia de un consenso en el seno de la comunidad científica no es incompatible con el disenso. Piénsese por ejemplo en las oposiciones entre escuelas, autores o modalidades de crítica. Todas ellas pueden estar en desacuerdo sobre las cuestiones que discuten, pero al menos tienen que estar de acuerdo para discutir sobre tales cuestiones y no sobre otras, lo que supone un acuerdo previo, de naturaleza no reflexiva, en torno al cual se definen los terrenos sobre los cuales debe discurrir el desacuerdo y los modos de expresión del desacuerdo.

23 "Décret relative à la licence es lettres. 8 juillet 1907” en Beauchamp (1909: 1007-1011).

24 Además de lo ya dicho en páginas anteriores, se puede añadir la influencia que los historiadores normaliens ejercieron en el entramado institucional, ya fuese en calidad de ministros (W. H. Waddington y A. Rambaud, a quienes se debe la creación de los puestos de maîtres de conférences y las becas de licenciatura y agregación, respectivamente) o como miembros destacados del aparato institucional (A. Dumont) o consejeros ministeriales, tal como sucedía con Lavisse, artífice de la reforma de la agrégation en historia de 1894, y Seignobos, eminencia gris de los programas escolares de 1902.Una visión más detenida en Noiriel (1990: 63-64), Gérard (en Amalvi et al, 2005: 285).

${ }^{25}$ Véase la "Circulaire relative à la licence es lettres. Décret du 8 juillet 1907” en Beauchamp 
la geografía, la ley de 1907 introduce medidas orientadas a favorecer -todavía más- la especialización histórica y el desarrollo de trabajos originales ${ }^{26}$.

2/ Dicho esto se puede finalizar el presente apartado con una reflexión sobre los efectos que tuvieron estas reformas para la creación de un espacio -a la vez lógico e institucional- llamado 'historia'. Lo más importante se ha dicho antes. $\mathrm{Si}$ algo caracteriza el desarrollo de las reformas universitarias es sin duda la conquista de una autonomía profesional (Noiriel, 1990: 61). En efecto, en apenas treinta años (1877-1907) la función y el estatus del historiador se han visto profundamente transformados. Ello es así, fundamentalmente, por efecto de una mutación de naturaleza colectiva, cuya extensión atañe no sólo al incremento de espacios dedicados a la enseñanza histórica, sino también al desarrollo y la incorporación de aquellos valores (metodológicos, deontológicos) que refuerzan el sentimiento de pertenencia a una colectividad. La institucionalización de la enseñanza histórica propicia así el desarrollo de una moral profesional, de un bagaje común basado en el cumplimiento de una trayectoria repleta de rituales y referencias compartidas por todos, profesores y alumnos: licences, jurados de agregación, seminarios impartidos por los maîtres de conférences, exámenes, libros canónicos, discursos pronunciados por los "padres fundadores" con motivo de la apertura del curso universitario ${ }^{27}$, etc. El resultado es una configuración cognitiva novedosa. Un espacio en el que una comunidad auto-delimitada de investigadores moviliza un conjunto de prácticas mediante las cuales se efectúa una selección organizada de la 'factualidad', con sus programas, sus objetos, sus evidencias procedimentales y toda una serie de inferencias y de analogías que reproducen un sentido específico (disciplinar) de la racionalidad (Fabiani en Revel et al, 2006: 11-34). Asimismo, se refuerza la idea de una objetividad que se desprende de la aplicación de las técnicas del medio, y para la cual la ciencia histórica establece su propia autonomía a condición de diferenciarse de otros saberes menos formalizados (textos de escritores, periodistas, nobles, bibliotecarios, etc.) y organizar su estructuración interna de acuerdo al control de los procedimientos enunciativos ${ }^{28}$.

Pese a ello, el éxito de la disciplina histórica resulta limitado. Es cierto que los avances fueron importantes; en apenas treinta años se había conquistado un espacio en el que un número creciente de personas (profesores y alumnos) trabajaban y discutían acerca de los mismos temas utilizando protocolos de aná-

(1909: 1045).

${ }^{26}$ Los cambios en las pruebas objetivas pueden verse en el "Décret relatif à la Licence es lettres. 8 juillet 1907' en Beauchamp (1909: 1008-1009).

27 Véanse los discursos dirigidos a los estudiantes de Lavisse (1885: 108-109; 126-127).

${ }^{28}$ El control de los procedimientos enunciativos supone una ruptura con la heterogeneidad procedimental que había caracterizado al saber histórico en la época anterior. Para entender esto es preciso tener en cuenta la pluralidad de procedencias socio-profesionales de quienes escribían libros de historia. La mayoría eran personas ajenas a la institución universitaria, fundamentalmente nobles o clérigos. Una muestra ilustrativa de datos para el periodo 1866-1876 en Carbonell (1976: 291-292). 
lisis (manipulativos, metodológicos, evaluativos) compartidos. Sin embargo, la fuerza de la disciplina histórica en su bastión parisino apenas podía emularse en otras plazas más periféricas de la geografía universitaria: por el momento, sólo la élite de los historiadores parisinos asemejaba la unidad de una corporación integrada. Posiblemente, por razones que tienen que ver con la homogeneidad de su reclutamiento institucional: casi todos ellos habían sido reclutados a través de criterios de admisión similares, sea por la École normale, sea por la agrégation en historia o bien por el doctorado en la Facultad de letras de la Sorbona (Noiriel 1990: 76).

Sea como fuere, la historia es, en este contexto, una disciplina fortalecida en el seno de la nueva universidad republicana, sobre todo en un lugar como París ${ }^{29}$, donde se concentran desde hace tiempo las instituciones dedicadas a la enseñanza, la investigación y la divulgación histórica (revistas, casas de edición, etc.).

Queda por ver sin embargo qué sucede con la sociología. ¿Existe alguna equiparación en términos institucionales? ¿De qué hablamos cuando hablamos de 'sociología' en este contexto?

\subsection{La sociología, dientes pequeños pero afilados...}

Para responder a estas preguntas hay que comenzar desmintiendo una falsa impresión, una idea que puede parecer razonable pero que no se sostiene a la luz de los análisis sobre la institucionalización académica de la sociología. En efecto, la palabra 'sociología' parecía estar en boca de la intelectualidad francesa de finales del siglo XIX. Durkheim había forjado sus reglas en 1895, y a ello le siguieron múltiples trabajos que hoy día siguen conservando la pátina de lo clásico. Tal época fue testigo además de la creación de los principales foros de difusión sociológica en Francia: la Revue internationale de Sociologie (1893), el Institut international de Sociologie (1894), la École des Hautes études sociales (1900-1910) y la célebre revista l'Année sociologique (1898), esta última tribuna desde la cual Durkheim y sus colaboradores (F. Simiand, P. Fauconnet, C. Bouglé, M. Mauss, etc.) lanzaban sus ataques contra los trabajos que no incorporaban las formas de inferencia de la sociología durkheimiana ${ }^{30}$. Por supuesto, tales instituciones no surgieron de la nada. A sus espaldas estaba toda la reserva de conocimientos empíricos que venían compilándose en materia de 'historia comparada' (gramática, historia de las religiones, historia de las instituciones) y

${ }^{29}$ De nuevo, los datos de Charles Olivier Carbonell (1976: 183-213) son contundentes.

${ }^{30}$ En verdad, la sociología hizo progresos semejantes en otros países europeos y en los Estados Unidos. En Italia, por ejemplo, se fundó la Rivista italiana de sociologia en 1897. En Alemania se fundó en 1888 el célebre Archiv für Sozialwissenschaft und Sozialpolitik, del cual M. Weber fue editor más tarde. En Gran Bretaña se creó la Sociological society en 1903 y en Bélgica el Institut de sociologie de Solvay en 1902. Por su parte, los Estados Unidos tampoco permanecieron al margen. En 1895 apareció el American Journal of Sociology, así como el célebre departamento de sociología de la Universidad de Chicago, centro de estudios sociológicos sobre urbanismo y criminología. 
ciencias humanas (etnología, demografía, economía, estadísticas), y con respecto a los cuales la sociología de Durkheim pretendía ser una suerte de guía o de autoconciencia crítica (Durkheim y Fauconnet 1903: 150).

En este sentido, la referencia a 'lo social' estaba presente en la vida intelectual francesa; de ella se hacían eco las publicaciones universitarias y las grandes discusiones de las ciencias humanas, razón por la cual Durkheim (1897: 7) se refería a la sociología como una palabra de moda a finales de siglo XIX. Sin embargo, la realidad distaba mucho de corroborar esta idea. En efecto, la sociología carecía en ese momento de una inserción disciplinar cualificada. Es verdad que se habían producido importantes reformas universitarias, pero ninguna de ellas implantó la sociología como una especialización científica o una licenciatura independiente. Su presencia se limitaba tan sólo a unos pocos cursos especializados o a un ámbito de discusión para-académico, en absoluto comparable al reconocimiento y los medios institucionales de los que gozaba la disciplina histórica en los primeros años del siglo XX.

En su origen, la enseñanza de la sociología se produjo de una manera encubierta, a través de una serie de cursos que trataban ciertas problemáticas (familia, derecho, pedagogía, religión, etc.) a la luz de una conceptualización nueva, de tipo sociológico. Por tanto, la sociología no existía como una disciplina autónoma, ni antes ni después de las priméras décadas del siglo xx. Sirvan pues como ejemplos los datos suministrados por Victor Karady (1976: 307) en su análisis sobre la estrategia de institucionalización universitaria desarrollada por los dukheimianos. En la mayor parte de las ocasiones se asiste a un balance negativo. El caso de Durkheim, por ejemplo, resulta especialmente revelador. A pesar de su valía intelectual, el padre fundador de la sociología obtuvo solamente una cátedra de pedagogía en 1902, lo que significa que jamás enseñó 'sociología' de un modo oficial y reconocido. Tampoco corrieron mejor suerte sus amigos de l'Année sociologique, quienes alcanzaron un reconocimiento bastante tardío de su valía intelectual. Maurice Halbwachs por ejemplo fue el primero en conseguir una cátedra de 'Sociología' en Francia, pero lo hizo en 1919, y en el seno de una universidad (Estrasburgo) alejada del núcleo parisino. Por su parte, F. Simiand obtuvo la cátedra de 'historia del trabajo' en el Collège de France en 1932, tres años antes de su fallecimiento. En todos estos casos el reconocimiento de la sociología se traducía en forma de cátedras independientes, nunca como una enseñanza instituida o una licenciatura diferenciada.

Más que como una disciplina en el plano institucional la sociología vincula su afirmación inicial con la emergencia de nuevos interrogantes y nuevas formas de inferencia (Revel: 1999: 390). Así pues, su éxito en el plano intelectual contrasta con el escaso papel que desempeña en las facultades universitarias. La presencia de la sociología seguía siendo testimonial. ¿Por qué? Para responder a esta pregunta es preciso subrayar el modo en que las reformas universitarias introdujeron las ciencias sociales (economía, geografía, sociología, etc.) en el ámbito de la enseñanza superior. ¿Qué lugar se atribuyó a estos saberes en un primer momento? ¿Detentaban una posición similar a la que ocuparon las 
disciplinas clásicas (historia, filosofía, lenguas, derecho...) o se les asignó una posición subalterna?

Como se sabe, las reformas universitarias marcaron un antes y un después en la enseñanza superior. Gracias a ellas se consiguió acabar con el retraso académico del viejo sistema napoleónico, modificándose la tipología de los cursos e introduciéndose una enseñanza basada en la innovación y la transmisión de los métodos de trabajo. Ahora bien, lejos de limitar su actividad a la reorganización de las disciplinas clásicas, las reformas propiciaron la irrupción de las ciencias sociales en el ámbito universitario, si bien es cierto que de una manera tenue e insinuada. Su emergencia apenas resultaba comparable con el peso y el vigor de las disciplinas clásicas (filosofía, lenguas clásicas, historia...) enseñadas en las facultades de letras; no obstante, poco a poco lograron introducirse por medio de la especialización de las cátedras existentes, lo cual propició una ocasión inmejorable para que los nuevos saberes pudieran infiltrarse en las aulas y los departamentos universitarios.

Así pues, la especialización de las disciplinas clásicas adquirió un grado considerable: se pasó de la impartición de clases genéricas al desarrollo de cursos cerrados y cátedras especializadas, acordes con los principios de clasificación contemporánea de las especialidades. Sin embargo, el caso de las ciencias sociales presentaba un esquema de especialización diferente, ya que no existía ningún itinerario previo sobre el cual pudiera continuarse una especialización progresiva. ¿Cómo introducir así los nuevos saberes? La estrategia seguía siendo la misma, si bien es cierto que la especialización se realizó sobre la base de las disciplinas clásicas existentes. Para ello era preciso estar en posesión de los títulos obtenidos en tales disciplinas (historia, filosofía, derecho, lenguas clásicas...), los únicos que existían en aquel momento. En este sentido, las ciencias sociales (geografía, economía, sociología) adoptaron una posición subalterna, ya que gran parte de sus principales valedores tuvieron que amoldar los nuevos conocimientos a los criterios y las expectativas que regían los programas de las facultades clásicas (derecho, letras). El caso paradigmático lo vemos en la trayectoria profesoral de Durkheim, quien tuvo que vincular su enseñanza sociológica a programas ajenos a la sociología propiamente dicha. Así, para justificar la utilidad universitaria de los nuevos saberes Durkheim se vio obligado, como tantos otros, a tratar las cuestiones del programa de agregación filosófica (Karady, 1979: 50-52) y vincular su enseñanza de la sociología con temáticas (pedagógicas) y facultades ajenas a las ciencias sociales.

Así, ante la pregunta por la inserción de los nuevos saberes, cabe responder lo siguiente: 'sí, las ciencias sociales se introdujeron en la universidad, pero lo hicieron de una manera subalterna y desigual, nunca bajo la forma de un itinerario especializado o una licenciatura independiente ${ }^{31}$. Con todo, el proceso exige

${ }^{31}$ Es verdad, como sostiene R. Ramos (1999: 12), que L. Liard mantuvo una confianza personal en la figura del joven Durkheim, pero esto no significaba que las ciencias sociales hubiesen adquirido una presencia estable e independiente en el ámbito universitario. No hay duda que la introducción de las ciencias sociales estuvo motivada por sectores del gobierno republicano; pero 
un examen diferenciado: hubo casos donde la inserción de las ciencias sociales se produjo prematuramente, como sucedía con la geografía $a^{32}$, pero hubo otros donde la implantación se dilató más en el tiempo, debido a las múltiples resistencias que tuvieron que lidiar con las disciplinas (mejor consolidadas) en las que alojaron originalmente sus cursos. El caso de la economía ${ }^{33}$ es claro al respecto, pero también el de la etnología, que ligó su afirmación disciplinar a instituciones localizadas de la enseñanza superior francesa, como la $5^{\text {a }}$ sección de la EPHE o ciertas cátedras en el Collège de France.

Lo mismo, mutatis mutandis, se puede decir de la enseñanza sociológica en las facultades de letras. Esta última accede al mundo universitario por medio de la especialización de las cátedras existentes, pero lo hace, eso sí, de manera desigual y deficitaria; en total, no se alcanzan siquiera la docena de cursos 'sociológicos' en la enseñanza superior francesa. Tanto es así que incluso se puede señalar el título de los mismos y el nombre de sus profesores. Entre los durkheimianos, cabe citar a Marcel Mauss y Henri Hubert, quienes ocuparon puestos en la $5^{\text {a }}$ sección de la EPHE impartiendo cursos como 'Historia de religiones de los pueblos no civilizados' y 'Religiones primitivas de Europa', respectivamente. Por su parte, Simiand hizo lo propio en la $4^{\mathrm{a}}$ sección de la EPHE con su seminario titulado 'Historia de los hechos y de las doctrinas económicas' (1912). Un poco más tarde, en 1919, Halbwachs consiguió la cátedra de 'Sociología y pedagogía' en la Universidad de Estrasburgo, y después, tras su paso por la Sorbona ingresó en el Collège de France durante los años 1944-1945.

En cuanto a los sociólogos no dukheimianos, se puede decir que monopolizaron las cátedras para-sociológicas en el Collège de France, vetando a Durkheim y a sus colaboradores hasta la década de 1930. De aquel grupo, cabe citar a Jean Izoulet, encargado de la cátedra de 'Filosofía social' (1897); Émile Levasseur, profesor de 'Geografía, Historia y estadísticas económicas' (1871); Georges Renard, titular de 'Historia del trabajo' (1907); Alfred Le Chatelier, profesor de 'Sociología y sociografía musulmanas' (1902); Édouard Fuster, encargado de 'Previsión y asistencia social' (1917) y Marcel Marion, titular del curso 'Hechos económicos y sociales' en 1912 (Karady 1976: 280-283). Por supuesto, esta lista no sería definitiva sin referenciar los cursos impartidos por Durkheim (1887-1901) durante su estancia en la universidad de Burdeos, en cuyo seno se asiste al despliegue de estadísticas y métodos de investigación sociológica, con el objeto de que el alumno se familiarice con las clasificaciones y las inferencia derivadas de las mediciones estadísticas (Ibíd: 279).

Tal situación permaneció estable hasta la Primera Guerra Mundial, lo que significa que la sociología ocupaba una posición subalterna en el conjunto del mercado universitario. No disponía en ese periodo (1890-1914) de ninguna titu-

tal empuje era todavía una apuesta tímida y no programada.

${ }_{32}$ Sobre la presencia subalterna de esta disciplina con respecto a la historia, véase Karady (1976: 275-277).

${ }^{33}$ La economía encontró sus condiciones de acceso al mercado universitario en las facultades de derecho. Para ello tuvo que adaptar su saber a las exigencias de los planes de estudios jurídicos. Véase Karady (1976: 283). 
lación o de diploma académico; todo lo más, un puñado de cátedras y de cursos anuales, pero en ningún caso un diploma que garantizase una profundidad en la materia. Dispersa por la geografía universitaria, la sociología se presenta como una disciplina auxiliar al servicio de otros saberes mejor consolidados. En tal sentido, tiene razón Jacques Revel cuando dice que la sociología vincula su aparición con la potencia de nuevos interrogantes. Al decir esto no trata sino de poner de manifiesto la enorme paradoja que suponía por un lado el reconocimiento intelectual de la sociología durkheimiana y su escasa presencia institucional por otro. Tal vez fuese así, como sostiene Ramón Ramos (1999: 29-30), debido a la falta de apoyos que su proyecto de autonomía disciplinar tuvo entre los funcionarios ministeriales: conviene recordar a este respecto que la enseñanza sociológica se introdujo con el objetivo de renovar los estudios sobre moral, pedagogía y ciencias de la educación. El gobierno republicano no trataba de alentar el desarrollo de una disciplina autónoma, como quería Durkheim, sino contribuir al desplazamiento de los discursos conservadores que dominaban el campo de la educación moral ${ }^{34}$.

En resumen, la primera forma de reconocimiento institucional de la sociología (1890-1914) pasaba por su capacidad de transformar a su favor el mercado de competencias universitarias. Se trataba, pues, de hacer valer el punto de vista sociológico en el interior de los dominios de los saberes consolidados (historia, economía, derecho, filosofía....). La trayectoria de Durkheim constituía así un ejemplo claro en este sentido. No bastaba pues con reclamar la autonomía de la disciplina sociológica; esencial era también ubicar esta enseñanza en el marco de los estudios objetivamente consolidados, a fin de convencer a sus representantes de la utilidad que poseían las herramientas sociológicas para la "mejora" de sus respectivos itinerarios (filosóficos, históricos, jurídicos). De ahí la compleja trayectoria profesoral de algunos sociólogos de la época: la única opción disponible pasaba por buscar un público variado de asistentes, lo cual exigía ligar su estrategia promocional a la búsqueda de alianzas con diferentes departamentos ya consolidados. Desde este punto de vista, la estimación durkheimiana de que la sociología es una ciencia en intersección con otras ciencias adquiere un significado no exento de cierta ambigüedad. Por un lado, se presenta como una proclama epistemológica, ya que sus defensores preconizaban la necesidad de transformar las ciencias sociales en ramas particulares de la sociología, pero por otro, se explica igualmente debido a una necesidad eminentemente práctica, sin la cual no hubiera sido posible la promoción interna de la disciplina. La socio-

${ }^{34}$ En este sentido, cabe cuestionar la tesis de Wolf Lepenies (1994: 39-47), según la cual la sociología adquiere carta de naturaleza en la Nouvelle Sorbonne. Tal interpretación, sustentada en las opiniones de los adversarios de Durkheim (Agathon, Charles Péguy), resulta incapaz de ofrecer una imagen fidedigna de la disciplina sociológica. Una cosa es admitir el influjo intelectual de Durkheim, otra confundir esta influencia con la institucionalización real de una disciplina en los planes de estudio. Que Durkheim fuese una suerte de "prefecto de estudios, soberano y autócrata" no quiere decir, sin embargo, que la sociología adquiriese "carta de naturaleza", ni mucho menos que la implantación de tales cursos fuese homogénea o se alinease en forma de diplomas o itinerarios específicos. 
logía necesitaba incrementar la demanda universitaria de sus servicios, para lo cual era preciso consolidar las alianzas y los servicios susceptibles de integrarla en el curso normal de otras disciplinas mejor consolidadas (Karady, 1979: 53).

\section{CONCLUSIÓN: EL DEBATE SIMIAND-SEIGNOBOS DESDE LA PERSPECTIVA DE LOS ENCLAVES DISCIPLINARES}

Llegados a este punto cabe explicitar un hecho que se ha sugerido en páginas anteriores: el debate no es una disputa entre iguales. Cierto es que la sociología cuenta con un mínimo de presencia universitaria, pero su posición es apenas relevante si se compara con el lugar que ocupa la ciencia histórica en el conjunto de la universidad republicana. Entre ambas disciplinas existe una relación asimétrica, lo cual permite comprender el carácter polémico que reviste algunas de las intervenciones, sobre todo por quienes no disponen de los recursos propios de la inserción universitaria.

En términos similares parece expresarse la historiadora Madeleine Rebérioux cuando se refiere a la célebre conferencia impartida por Simiand en 1903 (1983: 221). La sociología, dice, era una disciplina asemejada a una loba con dientes pequeños pero afilados, refiriéndose así a esa situación ambivalente según la cual la sociología era una disciplina marginal pero con muchas posibilidades de introducirse en el cerco universitario, en razón de la procedencia de sus valedores intelectuales -muchos de ellos formaban parte de la facción dominante (ENS) del establishment universitario- y de su decisión de utilizar esas posiciones para trasformar desde dentro la enseñanza de las disciplinas consolidadas, suscitando así una demanda creciente de instrucción y competencias sociológicas (Karady 1979: 53).

Sea como fuere, esta estrategia concierne al medio y largo plazo, pero no a la época en la que se desarrolló efectivamente el debate. En efecto, en ese momento la especialización de las cátedras implicaba todavía a pocos profesores, entre los cuales cabe citar, casi de manera exclusiva, a Durkheim y algunos pocos nombres más (H. Hubert, M. Mauss). El resto de durkheimianos eran todavía demasiado jóvenes u ocupaban posiciones subalternas en la institución universitaria. El caso de Simiand es claro al respecto: un joven, de apenas 30 años, cuya posición académica era prácticamente inexistente. Es más, la época en la que se desarrolla el debate (1903-1908) coincide con su etapa como bibliotecario del Ministerio de Comercio ${ }^{35}$. Es decir, aún no dispone de ninguna posición consolidada. Si su nombre en cambio adquiere relevancia es porque alberga buenas do-

35 A pesar de su juventud, Simiand gozaba de una formación intelectual envidiable: en 1896 había obtenido su agregación en Filosofía por la ENS. Después, alternó su trabajo en la biblioteca del Ministerio con la realización de una tesis doctoral en Derecho, algo que le proporcionó importantes conocimientos en materia económica y estadística. El título de su tesis ("Le Salaire des Ouvriers des Mines en France") era indicativo del horizonte en el que iba a moverse su producción intelectual. 
tes para la polémica científica. Como miembro de l'Année sociologique, Simiand realizó infinidad de recensiones sobre libros y otras publicaciones ${ }^{36}$. En algunos casos, con un afán crítico y metodológico, lo que suscitaba interesantes disputas con otras comunidades de investigación ${ }^{37}$.

Fiel al presupuesto de una ciencia social objetiva, Simiand no dudó en cuestionar los trabajos que justificaban sus resultados sobre la base de criterios internos a sus prácticas científicas. Si las ciencias humanas poseen un objeto común (el carácter 'social' de las realidades humanas) sus prácticas particulares (históricas, económicas, etnológicas, etc.) han de ser intercambiables, a fin de que los investigadores estén en condiciones de intercambiar y evaluar sus resultados de acuerdo a una epistemología común (Noiriel 2003: 52). Todo sucede como si la unidad del objeto (todas las ciencias humanas estudian en última instancia 'hechos sociales') justificase por sí sola la unidad del método científico: desde este punto de vista, no es necesario negar la pluralidad de las ciencias humanas, lo importante es que todas ellas sean comprendidas como ramas o modalidades particulares de una ciencia social que se rige por las reglas del método sociológico (Durkheim y Faucconnet 1903: 148).

He aquí el aspecto que subyace a las recensiones aparecidas en l'Année sociologique: concebir una ciencia social unitaria ${ }^{38}$, lo cual exige destituir la soberanía metodológica de las ciencias humanas e imponer en sus agendas el punto de vista sociológico. Ahora bien, de todas esas críticas merece la pena destacar el ataque realizado contra el método de los historiadores. Sea por su excelencia o su alto valor simbólico, la historia se perfila en ese momento como un blanco privilegiado y estratégico de ataque. Privilegiado porque la historia es un dispositivo disciplinar saturado de capital simbólico. Acumula en su seno un gran número de recursos objetivos, sea en forma de instituciones académicas (facultades, departamentos de historia, planes de estudio en secundaria, primaria), sea en forma de redes para-académicas (congresos, sociedades de historia, revistas, etc.), e incluso a través del capital simbólico que la ciencia histórica había cosechado en el conjunto de las instituciones políticas republicanas. En este sentido, atacar a la historia no es atacar a una rama cualquiera del saber. Es arremeter contra una de las disciplinas que gozaron de mayor reconocimiento en el seno de la enseñanza superior francesa, en razón de la trayectoria de sus representantes (algunos fueron ministros o colaboraron activamente en las reformas)

${ }^{36}$ Según Philippe Besnard, la contribución de Simiand a la revista es la siguiente: 139 reseñas de más de una página, 115 reseñas de menos de una página, 338 noticias de menos de 6 líneas, y 15 textos de introducción en secciones Todo ello le sitúa en cuarta posición tras la tríada Durkheim-Mauss-Hubert. Véase Besnard (en Guillard y Rosier 1996: 26).

37 Por ejemplo, con los geógrafos: "Géographie humaine et sociologie" (Simiand 1909: 723732). Pero también con los economistas: "Économie sociale" (Simiand 1905: 516-522). Y, por último, sus críticas a la sociología de cuño no durkheimiano, en especial a la obra de René Worms: "Organisme et société" (Simiand 1897a: 491-499), o la sociología de Marcel Bernes: "Sociologie et morale" (Simiand 1897b: 509-517).

${ }^{38}$ Sirva como pista el título de la intervención de Simiand en 1903. Allí se habla en todo momento en singular: "Método histórico y ciencia social". No se dice "ciencias sociales" en plural, sino ciencia social en singular. Véase Revel (1999: 391) 
y de su especial predilección por el estudio de la dimensión política del pasado, lo cual la convertía en la disciplina idónea para narrar la biografía de la nación.

Y estratégico porque al interpelar públicamente las debilidades de los historiadores, Simiand se posiciona en un marco en el que la exposición de sus recomendaciones cobra una resonancia ampliada, reclamando así de manera indirecta la necesidad de un espacio científico regido por las reglas del método sociológico. En efecto, si la historia no es un saber cualquiera, entonces todo posicionamiento que ponga al descubierto sus debilidades metodológicas se sitúa de inmediato en una posición intelectualmente privilegiada.

Ahora bien, ¿qué ocurre cuando esta interpelación se realiza desde posiciones con escasos recursos institucionales, como sucedía con la sociología? En este caso, diremos que el debate epistemológico se desdobla en una suerte de agresión disciplinar. El propósito de Simiand no es discutir simplemente las insuficiencias de los historiadores, sino tratar de convertir su ataque y su recomendación intelectual en una estrategia de promoción universitaria, a fin de que las reflexiones sociológicas puedan gozar de un territorio disciplinar diferenciado. Así, en ausencia de una verdadera implantación académica, la sociología encontraba en la disputa con los historiadores una ocasión magnífica para ensayar nuevas formas de legitimación disciplinar. Además de su vertiente metodológica, el debate esconde un ataque encubierto contra el influjo que los historiadores ${ }^{39}$ estaban ejerciendo en el ámbito de las ciencias humanas. Por eso creo que haríamos un mal servicio si agotásemos la lectura del debate en un episodio donde sólo se dirimen cuestiones de tipo metodológico o epistemológico: por debajo o por mediación de las recomendaciones se vislumbra una reacción de los durkheimianos ante la hegemonía ejercida $\left(\mathrm{y}\right.$ postulada ${ }^{40}$ ) por el dispositivo histórico en el conjunto de las ciencias humanas de la universidad republicana, a pesar de que tal propósito nunca aparezca como tal en el desarrollo de la disputa.

En efecto, si algo ha puesto de manifiesto el epígrafe anterior es sin duda la disparidad de condiciones en las que se encontraban los respectivos enclaves disciplinares de Simiand y Seignobos. Afirmar esto no es negar el valor o la legitimidad del debate epistemológico, es plantear la necesidad de articular la com-

${ }^{39}$ Nos referimos a la generación de historiadores normaliens que dominó el campo de la historiografía entre 1880 y 1930, es decir aquellos que ocuparon puestos en la administración pública y en los órganos universitarios: la presidencia y la asesoría en los ministerios de Instrucción Pública (W. H. Waddington, A. Rambaud), la dirección de las grandes écoles (A. Dumont, como director de la Escuela francesa de Atenas, G. Monod en la École Pratique des Hautes Études, etc.), la participación en las comisiones pedagógicas (Société pour l'étude des questions d'enseignement supérieur) que lucharon en favor de la reforma universitaria (Lavisse, Monod, Renan, Seignobos, Fustel de Coulanges), o la dirección de las grandes colecciones editoriales (Louis Halphen y Philippe Sagnac, Henri Berr, etc.).

${ }^{40}$ En su libro El método histórico aplicado a las ciencias sociales (1901), Ch. Seignobos defiende la idea de que la historia debe ejercer un papel rector sobre las ciencias sociales. Semejante posición constituye el detonante del debate, al considerar los durkheimianos que la propuesta del historiador francés no sólo reduce la investigación histórica a un procedimiento descriptivo sino que dota de su carácter heurístico al resto de las ciencias sociales. 
prensión de la disputa teórica con las condiciones reales en las que se desarrolla el ejercicio profesional de una comunidad de investigación.

Decía Pierre Bourdieu y Jean Claude Passeron (2005: 53) que los comienzos de las ciencias son momentos interesantes, ya que son indicadores de las circunstancias más propensas a explicitar los principios de construcción que caracterizan a una ciencia. En este sentido, el debate entre Seignobos y Simiand constituye un ejemplo privilegiado para ilustrar el modo en que dos comunidades investigadoras, cada una de ellas desigualmente equipadas en términos de capital simbólico e institucional, pugnan entre sí para imponer los principios de especificidad de sus objetos, así como para proclamar su papel rector en el concierto de las ciencias humanas de la universidad republicana.

Nada mejor pues que tal disputa para tomar conciencia de la situación asimétrica, profundamente desigual, desde la que la sociología francesa tuvo que conquistar su territorio, estabilizar su objeto y edificar nuevos modelos de causalidad. Así, en el debate que enfrenta a los sociólogos y los historiadores no se debería ver sólo una crítica de los determinismos implícitos del método histórico; por debajo o mediación de ello se perfila otra batalla, de naturaleza institucional, que opone dos candidaturas a la hegemonía de las ciencias humanas en Francia.

Naturalmente esta disputa se saldó con la victoria institucional de la ciencia histórica sobre la sociología, quizá porque la relación entre ambas era todavía demasiado asimétrica. No obstante, el ataque de los durkhemianos tampoco cayó en saco roto, ya que lo que comenzó siendo una querella entre disciplinas desiguales, propició a medio plazo un cambio en el espacio de la discusión historiográfica: de ahí en adelante muchos historiadores no pudieron permanecer indiferentes ante la ampliación de problemáticas que empezaban a insinuarse desde los márgenes del campo historiográfico (Henri Berr, Paul Mantoux) y las trincheras de la sociología durkheimiana: tratamientos formales de datos, periodizaciones de larga duración, descripciones multidimensionales de áreas de la cultura, etc. He aquí, precisamente, lo que sucede en el trascurso de los años posteriores, cuando Lucien Febvre y Marc Bloch recogen el testigo dejado por los durkheimianos y empiezan a implementar en sus agendas de trabajo las sugerencias de los sociólogos y los historiadores heterodoxos. El propio Bloch, en su Apología para la historia (2001: 49), expresaba su deuda con el fundador de l'Année sociologique de manera bastante gráfica; lo que le debe, dice, es haber aprendido "a analizar con mayor profundidad (...), a pensar (...) de manera menos barata". De ahí la importancia del presente debate para la historia de la historiografía y la epistemología de las ciencias sociales. En él se encuentran enunciadas las claves que van a guiar el programa de trabajo desarrollado después por la escuela de los Annales, cuyo resultado manifiesto será la reorganización interna de la ciencia histórica en Francia al calor del intercambio cruzado que la historia, la antropología y la sociología han realizado -no sin conflictos- de sus propiedades teóricas. 


\section{BIBLIOGRAFÍA}

AMALVI, Ch (dir.) (2005): Les Lieux de l'histoire, París, Armand Colin.

BESNARD, Ph. (1986): "L'impérialisme sociologique face a l'Histoire", en AAVV, Historiens et sociologues aujourd'hui, París, Éditions du CNRS, pp. 27-35.

BESNARD, Ph. (1996): "Le groupe durkheimien et le combat épistemologique pour la sociologie", en Guillard, L. y Rosier, M., eds., François Simiand (1873-1935), Amsterdam, Éditions des archives contemporaines, pp. 25-30.

BEAUCHAMP, A. (1880): Recueil des lois et réglements sur l'enseignement supérieur. Tome 1 (1789-1847), París, Delalain fréres.

BEAUCHAMP, A. (1909): Recueil des lois et réglements sur l'enseignement supérieur. Tome 6 (1898-1909)", París, Delalain fréres, 1909.

BOURDIEU, P., PASSERON, J. -C., CHAMBOREDON, J. -C. (2005): El oficio de sociólogo. Presupuestos epistemológicos, Madrid, Siglo XXI.

CARBONELL, Ch. -O. (1976): Histoire et historiens. Une mutation idéologique des historiens français 1865-1885, Toulouse, Privat.

CHARLE, Ch. (1994): La République des universitaires, París, Éditions du Seuil.

DELACROIX, C., DOSSE, F., GARCÍA, P., eds, (2007): Les courants historiques en France XIX-XX siécles, París, Folio-Gallimard.

DEVOTO, F. J. (1992): Entre Taine y Braudel. Itinerarios de la historiografía contemporánea, Buenos Aires, Editorial Biblos.

DURKHEIM, É. (1897), El suicidio, Madrid, Akal (2013).

DURKHEIM, É. y FAUCONNET, P. (1903): "Sociologie et sciences sociales", en Textes, París, Ed. de Minuit, 1975. pp. 121-159.

DURUY, V., eds, (1902): “Circulaire relative à la agrégation d'histoire (1894)", en Circulaires et instructions officielles relatives à l'instruction publique. Tome 12, Typ. de Delalain frères, Paris,, pp. 71-78.

GUILLARD et ROSIER, M (dir.): François Simiand (1873-1935), Amsterdam, Éditions des archives contemporaines.

JULLIAN, C. (1884): 'Notes sur les séminaires historiques et philologiques des universités allemandes $\bigotimes$, Revue internationale de l'enseignement, Tome 8, G. Masson éditeur, 1884, pp. 420-426.

KARADY, V. (1976): "Durkheim, les sciences sociales et l'Université: bilan d'un semi-échec", Revue française de Sociologie, 17-2, À propos de Durkheim, pp. 267311.

KARADY, V. (1979): "Stratégies de réussite et modes de faire-valoir de la sociologie chez les durkheimiens", Revue française de Sociologie, 20-1, Études et documents réunis par Philippe Besnard, pp. 49-82.»

KARADY V. (1983): “Les professeurs de la République. Le marché scolaire, les réformes universitaires et les transformation de la fonction professorale à la fin du XIX ${ }^{\mathrm{e}}$ siécle", Actes de la recherche en sciences sociales, Volume 47, 1983, pp. 90-112.

KEYLOR, W. R. (1975): Academy and Community. The foundation of the French Historical Profession, Harvard University Press. Cambridge, Massachusetts.

LAVISSE, E. (1881): "Le concours pour l'agrégation d'histoire et géographie", Revue internationale de l'enseignement supérieur. Tome 1, París, G. Masson Éditeur, pp. 137-151.

LAVISSE, E. (1885): Questions d'enseignement national, París, Armand Colin.

LEPENIES, W. (1995): Las tres culturas. La sociología entre la literatura y la ciencia, México, FCE. 
LOT, F. (1904): “L'Enseignement de l'histoire et de l'histoire de l'art dans les universités d'Allemagne et de France. Études statistiques", Bulletin de la Société d'Histoire moderne, 21 (février), pp. 114-121.

MONOD, G. (1876): "Du progrés des études historiques", Revue historique. Tome 1, París, Librairie Germer Bailliére, pp. 5-38.

MONOD, G. (1897): Portraits et souvenirs, París, Calmann Lévy éditeur.

NORIEL, G. (1990): “Naissance du métier d'historien”, Genèses, n¹, París, pp. 58-85.

NORIEL, G. (2003): "L'éthique de la discussion chez F. Simiand. A propos de deux conférences sur l'histoire (1903-1906)" en G. Noiriel, Penser avec, penser contre: itinéraire d'un historien, París, Belin, pp. 47-61.

PROST, A. (1968): Histoire de l'enseignement en France (1800-1967), París, Armand Colin.

RAMOS, R. (1999): La sociología de Émile Durkheim. Patología social, tiempo, religión, Madrid, CIS.

RÉBERIOUX, M. (1983): "Le débat de 1903: historiens et sociologues" en Au berceau des Annales. Le milieu strasbourgeois. L'histoire en France au début du XXe siècle, Toulousse, Presses de l'Institut d'études politiques de Toulousse.

REVEL, J. (1999): "Histoire et sciences sociales: lectures d'un débat français autour de 1900', en Hans Erich Bodeker, Peter Hanns Reill und Jurgen Schlumbo- hm, eds., Wissenschaft als kulturelle Praxis, 1750-1900, Gottingen, Van- denhoeck \& Ruprecht, p. 377-399.

SEIGNOBOS, Ch. (1881): "L'enseignement de l'histoire dans les Universités allemandes", Revue internationale de l'enseignement, Tome, 1, París, G. Masson Éditeur, pp. 565-600.

SEIGNOBOS, Ch. (1901): El método histórico aplicado a las Ciencias sociales, Madrid, Daniel Jorro, 1923.

SEIGNOBOS, Ch. (1907): "Les conditions pratiques de la recherche des causes dans le travail historique. Séance 30 mai 1907”, Bulletin de la société française de philosophie.7, París.

SEIGNOBOS, Ch. (1908): "L'inconnu et l'inconscient en histoire. Séance du 28 mai 1908", Bulletin de la société française de philosophie. 8, París, pp. 217-247.

SIMIAND, F. (1897a): "Organisme et société", Revue de Métaphysique et morale, París, Librairie Hachette, 1897, pp. 491-499.

SIMIAND, F. (1897b): "Sociologie et morale", Revue de Métaphysique et morale, París, Librairie Hachette, pp. 509-517.

SIMIAND, F. (1903): "Méthode historique et science sociale. Étude critique d'après les ouvrages récents de M. Lacombe et de M. Seignobos", en M. Cedronio, eds., Méthode historique et Sciences sociales, París, Éditions des Archives contemporaines.

SIMIAND, F. (1905): "Économie sociale", l'Année sociologique, Tome IX, Félix Alcan, Paris, pp. 516-522.

SIMIAND F. (1906): "La causalité en histoire. Séance du 31 mai 1906”, Bulletin de la Société française de philosophie, 6, París.

SIMIAND, F. (1909): "Géographie humaine et sociologie', l'Année sociologique, Tome XI, Félix Alcan, París, pp. 723-732 
